\title{
Thermotunable Terahertz Negative-Index Metamaterials with Dielectric Spheres Embedded in Semiconductor Host
}

\author{
Yu Chen $\left(\mathbb{D},{ }^{1}\right.$ Zhihao Chen $\left(\mathbb{D},{ }^{1}\right.$ Binyi Qin $\mathbb{D}^{\circ},{ }^{2}$ and Xiaoyu Dai $\mathbb{D}{ }^{1}$ \\ ${ }^{1}$ International Collaborative Laboratory of 2D Materials for Optoelectronics Science and Technology, \\ Key Laboratory of Optoelectronic Devices and Systems of Ministry of Education and Guangdong Province, \\ College of Optoelectronic Engineering, Shenzhen University, Shenzhen 518060, China \\ ${ }^{2}$ School of Electronics and Communication Engineering, Yulin Normal University, Yulin 537000, Guangxi, China \\ Correspondence should be addressed to Xiaoyu Dai; xiaoyudai@126.com
}

Received 4 June 2018; Accepted 13 August 2018; Published 20 September 2018

Academic Editor: Charles Rosenblatt

Copyright (C) 2018 Yu Chen et al. This is an open access article distributed under the Creative Commons Attribution License, which permits unrestricted use, distribution, and reproduction in any medium, provided the original work is properly cited.

A possibility to realize thermotunable isotropic negative-index metamaterials up to terahertz regime is theoretically investigated. The proposed composite metamaterials consist of dielectric spheres embedded randomly in a semiconductor host medium. As the variation in the intrinsic carrier density in InSb due to a variation in temperature thus changes the plasma frequency, we show theoretically the provided composite metamaterials whose index of refraction is thermally tunable and potentially less propagation loss. Furthermore, the effects of the radius of the dielectric sphere on the modulation frequency and bandwidth are discussed. Finally, we find that the design parameters for the composites can be scaled for application in the higher frequency regions.

\section{Introduction}

Negative-index metamaterials (NIMs), with simultaneously negative permittivity and permeability, were introduced by Veselago nearly forty years ago [1] and have recently received much attention in the literature owing to great potential for applications, such as perfect lens [2], optical cloaking [3], and perfect absorber [4]. Recently, metamaterials have also been used to regulate the light field [5-7]. There has been much progress in the development of the metamaterials (MMs) from the microwave frequencies to infrared and optical frequencies. In the microwave regime, the MMs can be constructed as a combined arrays consisting of metal wires and split-ring resonators [8]. MMs at the infrared and optical frequencies were accomplished with pairs of metal rods [9] and for the inverted system of pairs of dielectric voids in metal [10]. Most of the MMs designs are constructed by the metallic elements, which incur many drawbacks such as high loss, very narrow bandwidth, and highly anisotropic. An alternative scheme was proposed based on the resonance in individual nonmagnetic dielectric scatterer with very high permittivity [11-14]. However, dielectrics with extremely high permittivity also suffer from large damping. Recently, Seo et al. have shown that isotropic NIMs up to optical frequencies can be realized with dielectric spheres embedded randomly in a negative permittivity host (NPH) medium [15]. As a result, the DS/NPH shows more robust characteristics over the DS/DH in terms of fabrication tolerance, bandwidth, and propagation loss.

Most provided NIMs usually display a narrow operational bandwidth, and the operational frequency is not tunable. The tunable MMs are obviously very highly desired and potential expectations for future optical devices are, for example, spatial light modulators and tunable optical filters. There are various strategies reported for tuning the magnetic resonance of the permeability include electrical control [16, 17], magnetically control [18], and temperature changing $[19,20]$. Generally, tunability is introduced by ferromagnetic materials [21, 22], ferroelectric [13, 23], liquid crystal $[20,24,25]$, etc. However, the abovementioned tunable NIMs mostly operated at the low frequencies or high losses. In this paper, we will propose the designs of the thermotunable and low loss isotropic negative-index metamaterials up to terahertz region, which consists of 


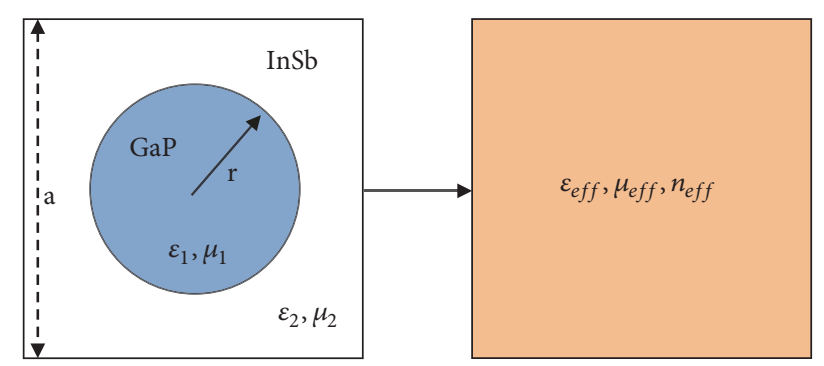

Figure 1: A schematic of a dielectric sphere is embedded into the semiconductor host. $r$ is the radius of the dielectric sphere and $a$ is the unit cell size.

dielectric spheres embedded randomly in a semiconductor host medium.

\section{The Permittivity of the Semiconductor and the Effective Medium Theory}

In the terahertz regime, the complex-valued relative permittivity of InSb is given by the simple Drude model [26]

$$
\varepsilon(\omega)=\varepsilon_{\infty}-\frac{\omega_{p}^{2}}{\left(\omega^{2}+i \gamma \omega\right)},
$$

where $\omega$ is the angular frequency, $\varepsilon_{\infty}$ represents the highfrequency permittivity,and $\gamma$ is the damping constant; $\omega_{p}$ is the plasma frequency and $\omega_{p}=\sqrt{N e^{2} / \varepsilon_{0} m^{*}}$ depends on the intrinsic carrier density $N$, the effective mass $m^{*}$ of free carriers, the electronic charge $e$, and the free-space permittivity $\varepsilon_{0}$. Compared to metals, the plasma frequency $\omega_{p}$ of InSb depends strongly on the temperature $T$. The intrinsic carrier density $N\left(\right.$ in $^{-3}$ ) in InSb obeys the relationship [27]

$$
N=5.76 \times 10^{20} T^{3 / 2} \exp \left(-\frac{0.26}{2 k_{B} T}\right),
$$

where $k_{B}$ is the Boltzmann constant and the temperature is in Kelvin. A variation in $N$ due to a variation in $T$ thus changes $\omega_{p}$. Consequently, in the far-infrared portion of the terahertz regime, $\varepsilon(\omega)$ of InSb is very sensitive to T. Hence, for NIMs comprising InSb host and dielectric sphere, we can expect that temperature variations can cause substantial variations in the optical response characteristics.

The composite material consisting of spherical scatters can be described as a homogeneous medium in the subwavelength limit and the effective electric permittivity and effective magnetic permeability are $\varepsilon_{e f f}(\omega)$ and $\mu_{e f f}(\omega)$, respectively. As shown in Figure 1, we assume that the dielectric spheres possessing a relative dielectric permittivity $\varepsilon_{1}$ and relative magnetic permeability $\mu_{1}$ are embedded in a semiconductor host medium whose relative permittivity and relative permeability are $\varepsilon_{2}(\omega)$ and $\mu_{2}=1$, respectively. Assuming that the dielectric spheres are located in the host medium randomly and homogeneously, in the quasistatic limit, we can derive the relative effective permittivity $\varepsilon_{e f f}(\omega)$ and the relative effective permeability $\mu_{e f f}(\omega)$ from the extended Maxwell-Garnett theory (EMG), as shown in [14]

$$
\begin{aligned}
& \varepsilon_{e f f}(\omega)=\varepsilon_{2} \frac{x^{3}-3 i f T_{1}^{E}}{x^{3}+(3 / 2) i f T_{1}^{E}} \\
& \mu_{e f f}(\omega)=\mu_{2} \frac{x^{3}-3 i f T_{1}^{H}}{x^{3}+(3 / 2) i f T_{1}^{H}}
\end{aligned}
$$

where $f$ is the volume fraction representing the ratio of the volume of the dielectric spheres to the total volume and $f=(4 / 3) \pi r^{3} / a^{3}$, where $r$ denotes the sphere radius and $a$ is the unit cell size. $T_{1}^{E}$ and $T_{1}^{H}$ are the electric-dipole and magnetic-dipole components of the scattering $\mathrm{T}$ matrix of a single sphere, respectively. They can be written as

$$
\begin{aligned}
& T_{1}^{E}(\omega) \\
& =\left[\frac{j_{1}\left(x_{1}\right)\left[x_{2} j_{1}\left(x_{2}\right)\right]^{\prime} \varepsilon_{1}-j_{1}\left(x_{2}\right)\left[x_{1} j_{1}\left(x_{1}\right)\right]^{\prime} \varepsilon_{2}}{h_{1}^{+}\left(x_{2}\right)\left[x_{1} j_{1}\left(x_{1}\right)\right]^{\prime} \varepsilon_{2}-j_{1}\left(x_{1}\right)\left[x_{2} h_{1}^{+}\left(x_{2}\right)\right]^{\prime} \varepsilon_{1}}\right], \\
& T_{1}^{H}(\omega) \\
& =\left[\frac{j_{1}\left(x_{1}\right)\left[x_{2} j_{1}\left(x_{2}\right)\right]^{\prime} \mu_{1}-j_{1}\left(x_{2}\right)\left[x_{1} j_{1}\left(x_{1}\right)\right]^{\prime} \mu_{2}}{h_{1}^{+}\left(x_{2}\right)\left[x_{1} j_{1}\left(x_{1}\right)\right]^{\prime} \mu_{2}-j_{1}\left(x_{1}\right)\left[x_{2} h_{1}^{+}\left(x_{2}\right)\right]^{\prime} \mu_{1}}\right],
\end{aligned}
$$

where $j_{1}$ is the spherical Bessel function and $h_{1}^{+}$is the spherical Hankel function for $l=1$, respectively. $[X(x)]^{\prime}=$ $d X / d x . x_{1}$ and $x_{2}$ stand for the sphere size parameter, $x_{1}=$ $(\omega / c) r \sqrt{\varepsilon_{1} \mu_{1}}=2 \pi r / \lambda_{1}$ and $x_{2}=(\omega / c) r \sqrt{\varepsilon_{2} \mu_{2}}=2 \pi r / \lambda_{2}$, and $\lambda_{1}$ and $\lambda_{2}$ are the wavelengths in the sphere medium and host medium, respectively.

\section{Numerical Results and Discussion}

Figure 2 shows the simulation results of $\varepsilon_{\text {eff }}, \mu_{\text {eff }}, n_{\text {eff }}$ for $T=$ $220 \mathrm{~K}, f=0.2$, and $r=60 \mathrm{um}$ using [3-5]; here we have assumed that $\varepsilon_{\infty}=15.68, m^{*}=0.015 m_{e} \mathrm{~kg}, m_{e}=9.1 \times$ $10^{-31}$, and $\gamma=2 \pi \times 0.05 \mathrm{THz}$ [27], and the gallium phosphide $(\mathrm{GaP})$ is assumed for the nonmagnetic dielectric sphere, and the permittivity $\varepsilon_{1}=12.25 \times\left(1+i \times 10^{-3}\right)$.

We can see from the figures that the real part of the effective permittivity $\varepsilon_{e f f}$ is negative from $0.58 \mathrm{THz}$ to 0.9 $\mathrm{THz}$, and the real part of the effective permeability $\mu_{\text {eff }}$ is negative from $0.65 \mathrm{THz}$ to $1.0 \mathrm{THz}$; hence the double region is between about $0.65 \mathrm{THz}$ and $0.9 \mathrm{THz}$ and the bandwidth is about $0.25 \mathrm{THz}$, as shown in the Figure 2(c). Also we can find that the value of $\varepsilon_{e f f}$ is far larger than $\mu_{e f f}$; however the imaginary parts of $\varepsilon_{e f f}, \mu_{\text {eff }}, n_{\text {eff }}$ are all much smaller than the composite MMs constructed by the metallic sphere when the incident wave frequency is far away from the resonance regions.

In order to discuss the effects of the temperature $T$ on the properties of $\varepsilon_{e f f}, \mu_{e f f}, n_{e f f}$, in Figure 3, we have plotted the frequency-dependent refractive-index of composite MMs at various temperatures. We can find from Figure 3(a) that the resonance dips are almost kept fixed as the temperature increases; however, the value of the real part 


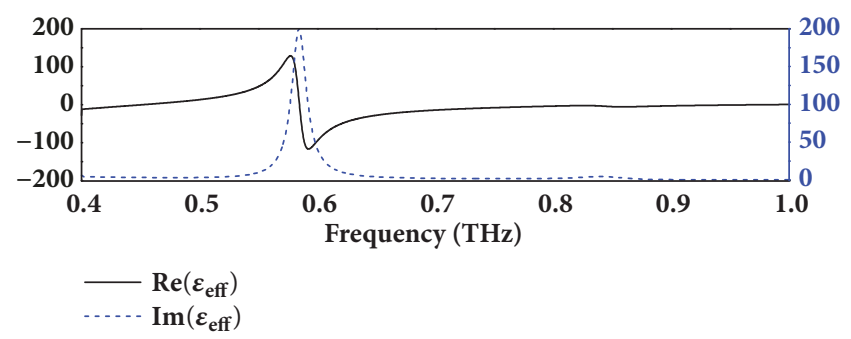

(a)

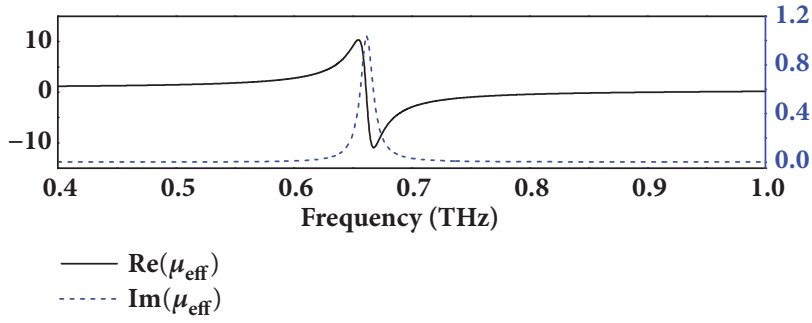

(b)

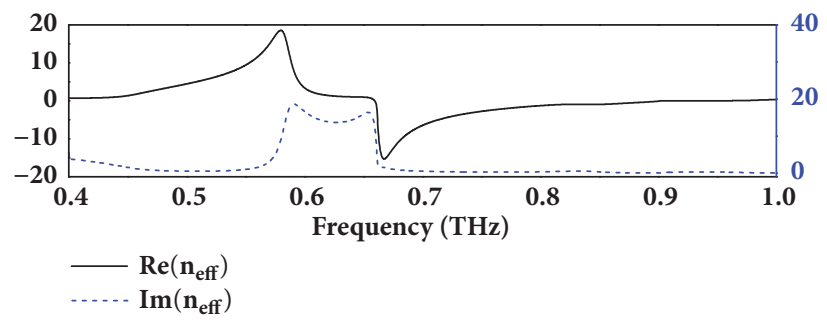

(c)

FIgURE 2: The numerical results of the real part (Re) and imaginary (Im) part of $\varepsilon_{e f f}, \mu_{e f f}, n_{e f f}$ of the composite DS/NPH effective metamaterials.

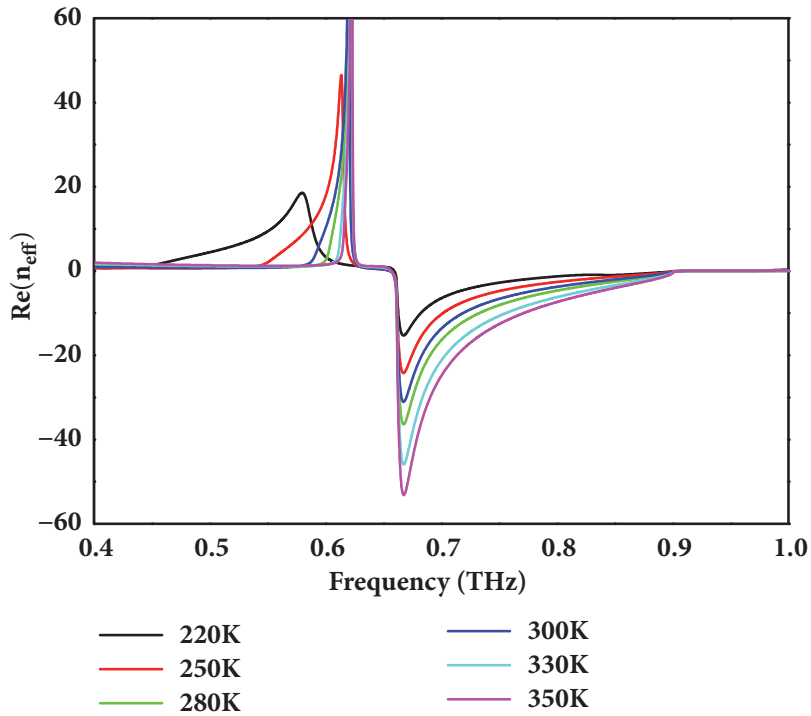

(a)

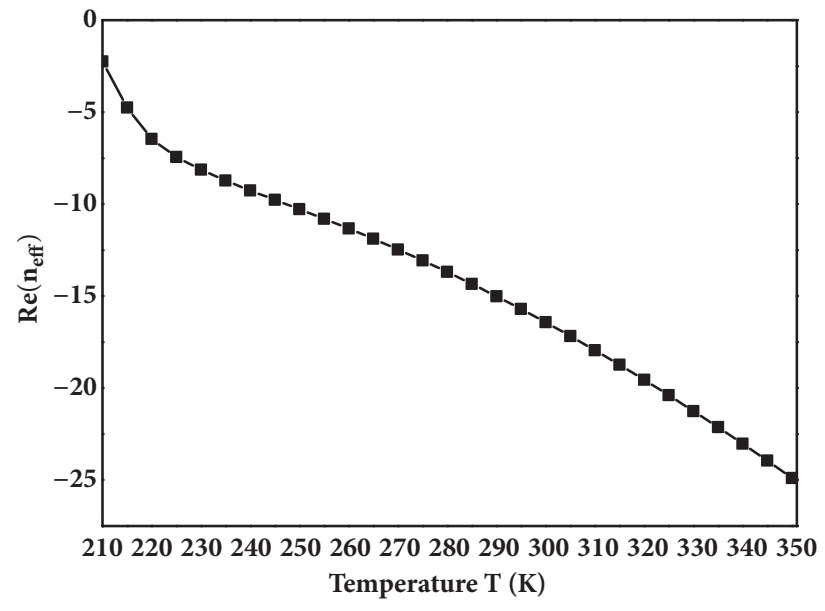

(b)

FIgURE 3: The thermotunable negative-index metamaterials. (a) The real part of the refractive-index $n_{\text {eff }}$ at different temperatures ranging from 220 to $350 \mathrm{~K}$. (b) The real part of the refractive-index $n_{\text {eff }}$ at $\omega / 2 \pi=0.7 \mathrm{THz}$ for different temperatures ranging from 210 to $350 \mathrm{~K}$.

of $n_{\text {eff }}$ will become bigger and bigger as the temperature increases. Clearly, the optical properties of the MMs, such as $\varepsilon_{e f f}, \mu_{e f f}, n_{e f f}$, all change significantly with temperature. For proving these properties clearly, in Figure 3(b), we have shown the real part of $n_{e f f}$ as a function of temperature from $210 \mathrm{~K}$ to $350 \mathrm{~K}$. As expected, $n_{\text {eff }}$ can be significantly enlarged by raising the temperature. The temperature-dependent optical properties of composite MMs can be attributed directly to the thermally sensitiveness of the plasma frequency for the permittivity of the semiconductor InSb.
Further, we find that the properties of $\varepsilon_{e f f}, \mu_{e f f}, n_{e f f}$ in our composite structure depend on the radius of the dielectric sphere strongly; hence the spectral position of the resonance point can be tuned by varying the radius of the dielectric sphere. For proving this, we have shown the dependence of the real part of $n_{\text {eff }}$ on the radius of the dielectric sphere in Figure 4. It is shown that when the radius of the sphere changes from $50 \mathrm{um}$ to $80 \mathrm{um}$, the resonance frequency points of the negative refractive-index will move from 0.8 $\mathrm{THz}$ to $0.5 \mathrm{THz}$ and the value of the resonance negative 


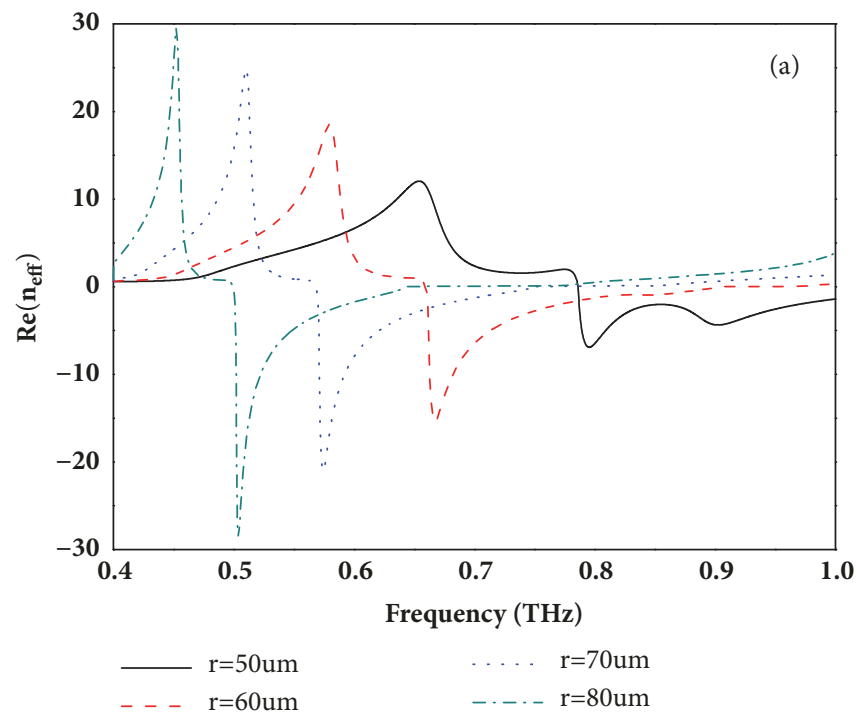

FIGURE 4: The real part of $n_{e f f}$ as a function of the radius of the dielectric sphere, where $f=0.2$ and $T=220 \mathrm{~K}$.

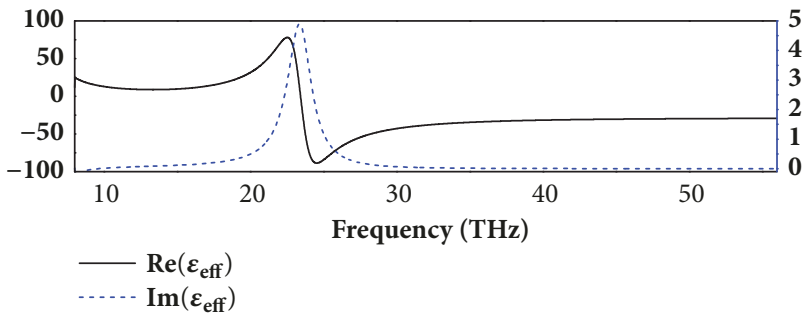

(a)

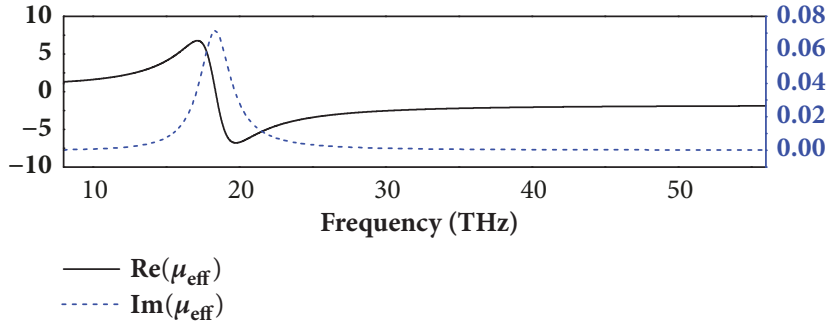

(b)

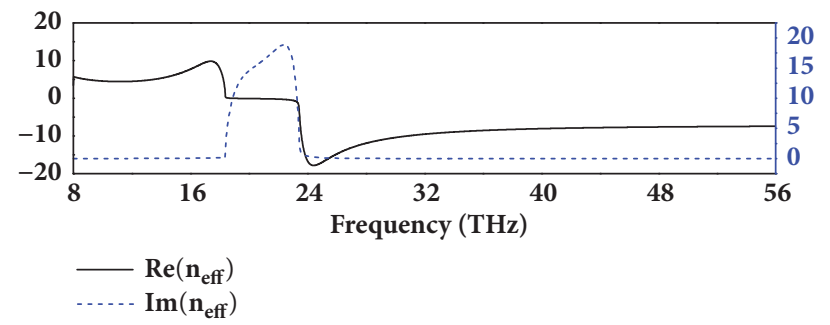

(c)

FIGURE 5: Calculated real and imaginary part of the complex permittivity, permeability, and refractive-index of the composite MMs in the terahertz region between 8 and $56 \mathrm{THz}$. Where $r=1 \mathrm{um}$ and $T=350 \mathrm{~K}$, the other parameters are same as in Figure 2 .

refractive-index point will become larger and larger as the radius of the dielectric sphere increases.

We have shown that the low loss and thermotunable NIMs can be realized at the terahertz frequencies; however the design of the material is scalable within a large dynamic range of operating frequencies as long as we change the radius of the dielectric sphere and the temperature simultaneity. First, the intrinsic carrier density in InSb can be changed from $N=1.977 \times 10^{21} \mathrm{~m}^{-3}$ at $220 \mathrm{~K}$ to $N=5.065 \times 10^{22}$ at $350 \mathrm{~K}$, which will lead to the plasma frequency of the semiconductor host altering from $\omega_{p}=2.046 \times 10^{13}$ at $220 \mathrm{~K}$ to $\omega_{p}=1.036 \times$ $10^{14}$ at $350 \mathrm{~K}$; therefore if only we adjust the scale of the radius of the dielectric sphere, we can obtain the double negative metamaterials at higher frequency. Figure 5, for example, shows the corresponding real part of the refractive-index from $8 \mathrm{THz}$ to $56 \mathrm{THz}$, where $r=1 \mathrm{um}$ and $T=350 \mathrm{~K}$, the other parameters are the same as in Figure 2. Again, it clearly demonstrates that one can obtain a bulk material of double negative metamaterials by embedding the dielectric spheres in a semiconductor host medium randomly at the terahertz even higher frequency. Moreover, the frequency bandwidth also becomes very large to reach $32 \mathrm{THz}$.

\section{Conclusions}

In conclusion, we have provided a scheme to realize a thermotunable and less loss isotropic negative-index metamaterials 
up to terahertz regime by embedding the dielectric spheres in a semiconductor host medium randomly. The composite metamaterial is sensitive to the outer temperature, which makes the double negative frequency regions tunable. Furthermore, the radius of the dielectric sphere will also make the frequency ranges of the double regions tunable. Moreover, by scaling the size of the spheres and outer temperature, we can vary the operating frequency to higher frequency. Such reconfigurable and thermotunable metamaterials have very promising future for designing tunable devices, such as tunable optical cloaking, tunable spatial light modulators, and tunable novel microwave antenna.

\section{Data Availability}

The [DATA TYPE] data used to support the findings of this study are available from the corresponding author upon request.

\section{Conflicts of Interest}

The authors declare that they have no conflicts of interest.

\section{Acknowledgments}

This work is supported by the National Natural Science Foundation of China (Grants nos. 61505122, 61505124), the Natural Science Foundation of SZU (Grant nos. 2016031, 2017018), the program of Fundamental Research of Shenzhen Science and Technology Plan (Grant no. JCYJ20160422152152634), the project supported by Guangdong Natural Science Foundation (Grant no. 2016A030310065), the Natural Science Foundation Guangdong Education Department (2015KQNCX146), and Yulin Normal University Research Grant (Grant no. 2016YJKY06).

\section{References}

[1] V. G. Veselago, "The electrodynamics of substances with simultaneously negative values of and $\mu$," Soviet Physics Uspekhi, vol. 10, no. 4, pp. 509-514, 1968.

[2] J. B. Pendry, "Negative refraction makes a perfect lens," Physical Review Letters, vol. 85, no. 18, pp. 3966-3969, 2000.

[3] D. Schurig, J. J. Mock, B. J. Justice et al., "Metamaterial electromagnetic cloak at microwave frequencies," Science, vol. 314, no. 5801, pp. 977-980, 2006.

[4] R. Alaee, M. Farhat, C. Rockstuhl, and F. Lederer, "A perfect absorber made of a graphene micro-ribbon metamaterial," Optics Express, vol. 20, no. 27, pp. 28017-28024, 2012.

[5] J. Li, T. Tang, L. Luo, N. Li, and P. Zhang, "Spin Hall effect of reflected light in dielectric magneto-optical thin film with a double-negative metamaterial substrate," Optics Express, vol. 25, no. 16, pp. 19117-19128, 2017.

[6] T. Tang, J. Li, Y. Zhang, C. Li, and L. Luo, "Spin Hall effect of transmitted light in a three-layer waveguide with lossy epsilonnear-zero metamaterial," Optics Express, vol. 24, no. 24, pp. 28113-28121, 2016.

[7] S. Liu, T. Cui, Q. Xu et al., "Anisotropic coding metamaterials and their powerful manipulation of differently polarized terahertz waves," Light Science \& Applications, vol. 5, no. 5, Article ID e16076, 2016.

[8] R. A. Shelby, D. R. Smith, and S. Schultz, "Experimental verification of a negative index of refraction," Science, vol. 292, no. 5514, pp. 77-79, 2001.

[9] A. Moreau, C. Ciracì, J. J. Mock et al., "Controlled-reflectance surfaces with film-coupled colloidal nanoantennas," Nature, vol. 491, no. 7427, pp. 86-89, 2012.

[10] S. Yang, X. Ni, B. Kante et al., "Experimental Demonstration of Optical Metamaterials with Isotropic Negative Index," in Proceedings of the Conference on Lasers and Electro-Optics, pp. 1-2, San Jose, California, 2016.

[11] Y. Ma, H. Zhang, Y. Li, and Y. Wang, "Miniaturized and dualband metamaterial absorber with fractal Sierpinski structure," Journal of the Optical Society of America B: Optical Physics, vol. 31, no. 2, pp. 325-331, 2014.

[12] W. Walasik, "Influence of the Filling Factor and Size of Coated Spheres on Effective Refractive Index in Nanosphere Dispersed Liquid Crystal Metamaterial," Nonlinear Optics Quantum Optics, vol. 44, no. 44, p. 129, 2012.

[13] M. V. Rybin, K. B. Samusev, and M. F. Limonov, "Multiscale modeling of all-dielectric metamaterials," in Proceedings of the 2015 Days on Diffraction (DD), pp. 1-5, St.Petersburg, Russia, May 2015.

[14] J. Li, Y. Xuan, and Q. Li, "Analysis of influence factors of negative refraction phenomenon in particulate composite," Science China Technological Sciences, vol. 55, no. 6, pp. 17191724, 2012.

[15] B.-J. Seo, T. Ueda, T. Itoh, and H. Fetterman, "Isotropic left handed material at optical frequency with dielectric spheres embedded in negative permittivity medium," Applied Physics Letters, vol. 88, no. 16, 2006.

[16] S. Qiao, Y. Zhang, S. Liang et al., "Multi-band terahertz active device with complementary metamaterial," Journal of Applied Physics, vol. 118, no. 12, p. 123106, 2015.

[17] T. H. Hand and S. A. Cummer, "Frequency tunable electromagnetic metamaterial using ferroelectric loaded split rings," Journal of Applied Physics, vol. 103, no. 6, 2008.

[18] S.-C. Zhao, X.-F. Qian, Y.-P. Zhang, and Y.-A. Zhang, "Negative refraction with little loss manipulated by the voltage and pulsed laser in double quantum dots," Progress of Theoretical and Experimental Physics, vol. 128, no. 2, pp. 243-250, 2012.

[19] W. Lewandowski, M. Fruhnert, J. Mieczkowski, C. Rockstuhl, and E. Górecka, "Dynamically self-assembled silver nanoparticles as a thermally tunable metamaterial," Nature Communications, vol. 6, 2015.

[20] S. T. Bui, V. D. Nguyen, X. K. Bui et al., "Thermally tunable magnetic metamaterials at THz frequencies," Journal of Optics, vol. 15, no. 7, p. 075101, 2013.

[21] B. Du, Z. Xu, J. Wang, and S. Xia, "Magnetically tunable ferritedielectric left-handed metamaterial," Progress in Electromagnetics Research C, vol. 66, pp. 21-28, 2016.

[22] K.-M. Li, C. Sun, and J.-S. Li, "Magnetically tunable terahertz wave left-handed material," in Proceedings of the Laser and TeraHertz Science and Technology, LTST 2012, China, November 2012.

[23] M. Gil, C. Damm, A. Giere et al., "Electrically tunable splitring resonators at microwave frequencies based on bariumstrontium-titanate thick films," IEEE Electronics Letters, vol. 45, no. 8, pp. 417-418, 2009. 
[24] M. Maasch, M. Roig, C. Damm, and R. Jakoby, "Planar metamaterial with separately voltage tunable electric and magnetic dispersion based on liquid crystal," in Proceedings of the 2013 7th International Congress on Advanced Electromagnetic Materials in Microwaves and Optics, METAMATERIALS 2013, pp. 556559, France, September 2013.

[25] P. Yaghmaee, O. H. Karabey, B. Bates, C. Fumeaux, and R. Jakoby, "Electrically tuned microwave devices using liquid crystal technology," International Journal of Antennas and Propagation, vol. 2013, Article ID 824214, 9 pages, 2013.

[26] S. C. Howells and L. A. Schlie, "Transient terahertz reflection spectroscopy of undoped InSb from 0.1 to $1.1 \mathrm{THz}$," Applied Physics Letters, vol. 69, no. 4, pp. 550-552, 1996.

[27] P. Halevi and F. Ramos-Mendieta, "Tunable photonic crystals with semiconducting constituents," Physical Review Letters, vol. 85, no. 9, pp. 1875-1878, 2000. 

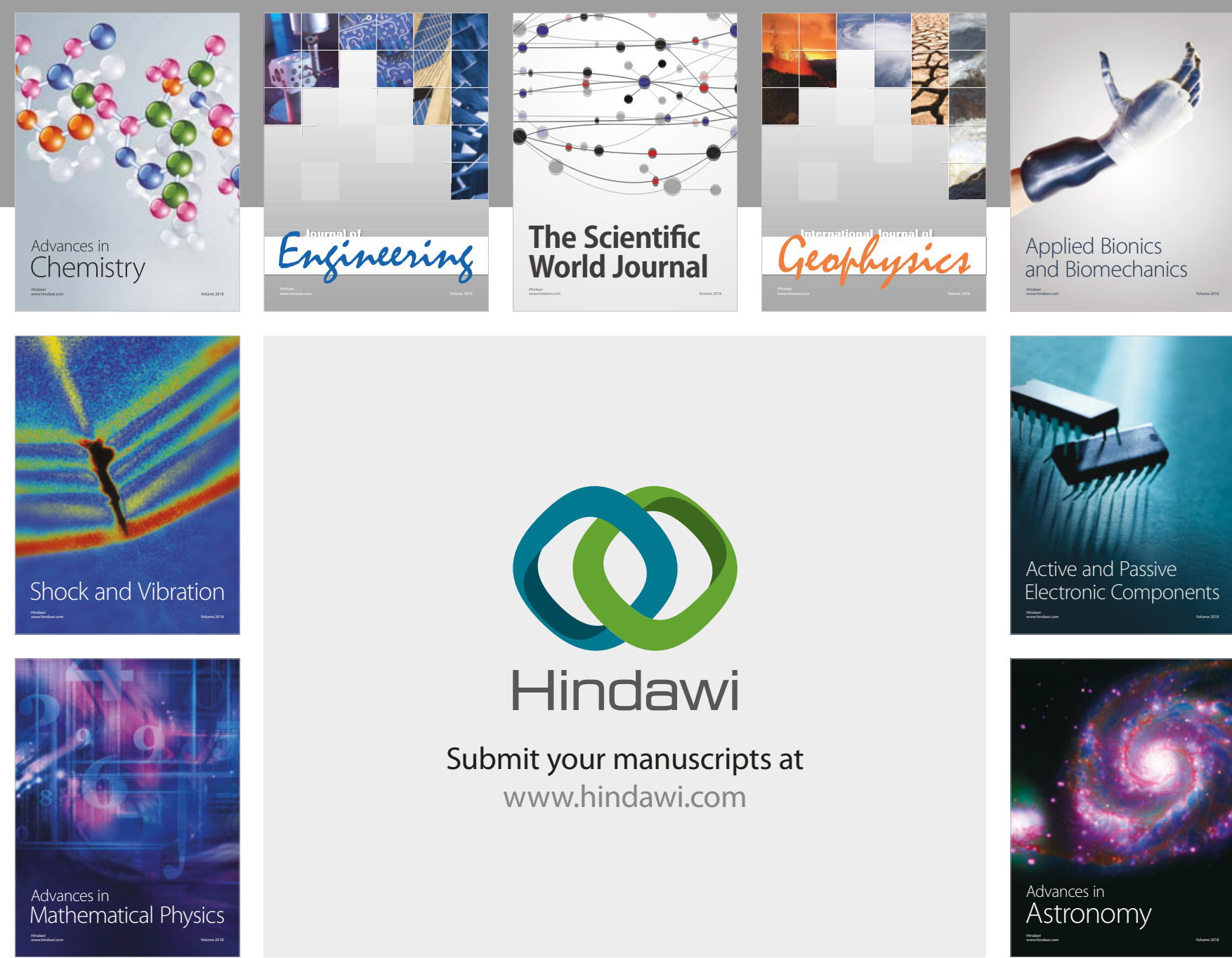

Submit your manuscripts at

www.hindawi.com

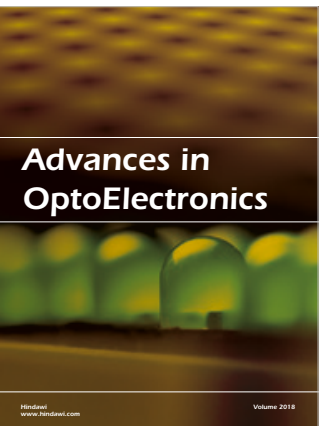

\section{Rotcting Machinery}
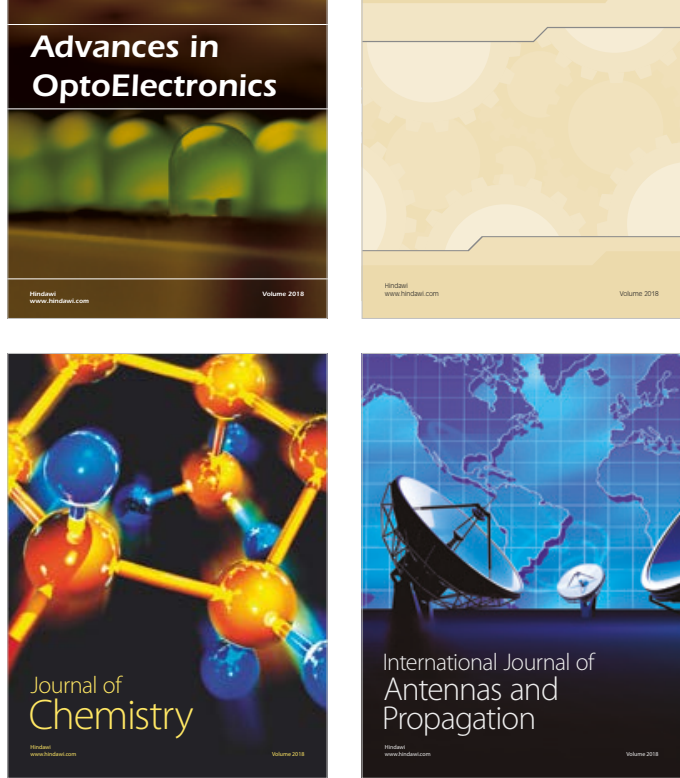

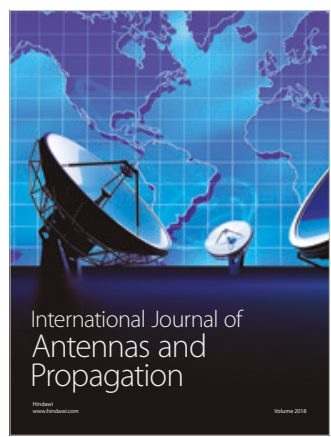

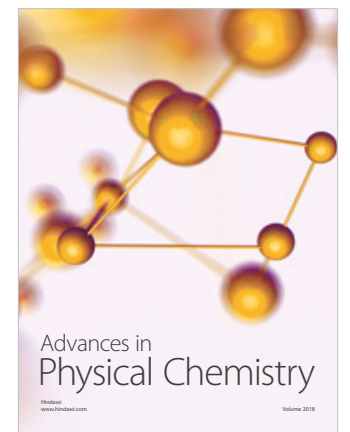

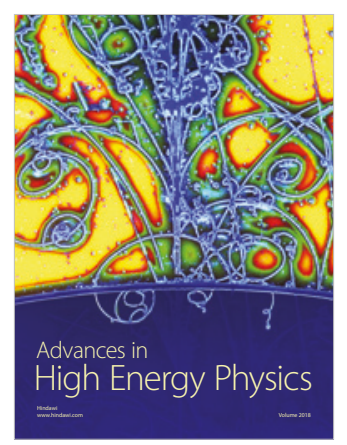

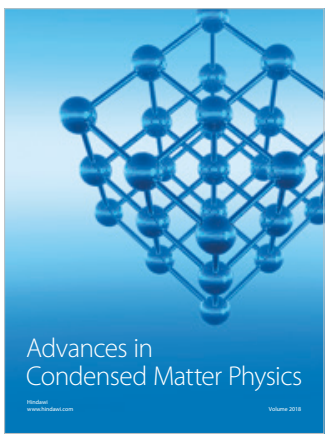

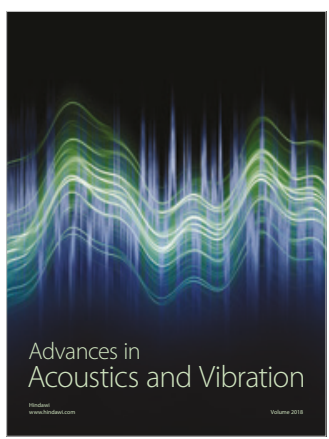

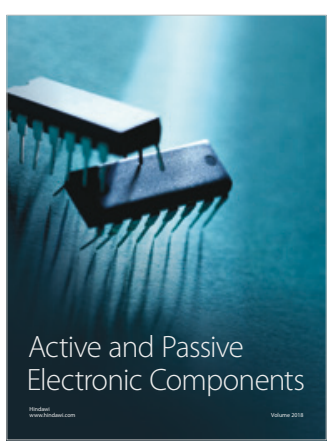
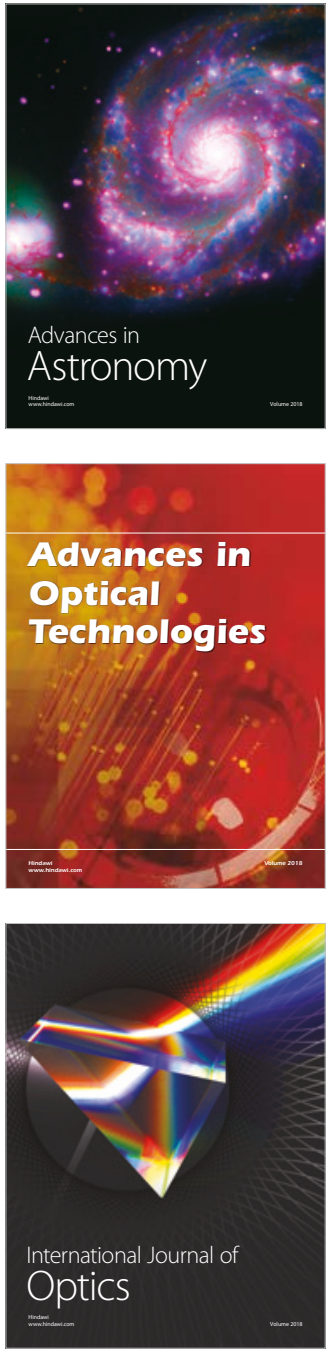